\begin{tabular}{|c|l|}
\hline Title & $\begin{array}{l}\text { Use of Implanted Markers and Interportal A djustment With Real-Time Tracking Radiotherapy System to Reduce } \\
\text { Intrafraction Prostate Motion }\end{array}$ \\
\hline Author(s) & $\begin{array}{l}\text { Shimizu, Shinichi; O saka, Y asuhiro; Shinohara, Nobuo; Sazawa, A taru; Nishioka, Kentaro; Suzuki, Ryusuke; Onimaru, } \\
\text { Rikiya; Shirato, Hiroki }\end{array}$ \\
\hline Citation & $\begin{array}{l}\text { International Journal of Radiation Oncology*Biology*Physics, 81(4), e393 e399 } \\
\text { https://doi.org/40.1016/.ijrobp.2011.04.043 }\end{array}$ \\
\hline Issue Date & 2011-11-15 \\
\hline Doc URL & http://hdl.handle.net/2115/50755 \\
\hline Type & article (author version) \\
\hline File Information & IJROBP81-4_e393_e399.pdf \\
\hline
\end{tabular}

Instructions for use 


\section{USE OF IMPLANTED MARKERS AND INTERPORTAL ADJUSTMENT WITH \\ REAL-TIME TRACKING RADIOTHERAPY (RTRT) SYSTEM TO REDUCE INTRA-FRACTION PROSTATE MOTION}

Running title: Reduction of Intra-fraction motion

Shinichi Shimizu, M.D. ${ }^{*}$, Yasuhiro Osaka, M.D. ${ }^{*}$, Nobuo Shinohara, M.D. ${ }^{\dagger}$, Ataru Sazawa, M.D. ${ }^{\dagger}$, Kentaro Nishioka, M.D. ", Ryusuke Suzuki, Ph.D. ${ }^{\ddagger}$, Rikiya Onimaru, M.D. ", Hiroki Shirato, M.D.

"Department of Radiation Medicine, Hokkaido University School of Medicine, Sapporo, Japan;

'Department of Urology, Hokkaido University School of Medicine, Sapporo, Japan;

‡Department of Medical Physics, Hokkaido University School of Medicine, Sapporo, Japan

Correspondence: Shinichi Shimizu, M.D. Department of Radiology, Hokkaido University School of Medicine, North-15 West-7, Kita-ku, Sapporo, Japan. Tel: 81-11-716-1161, Fax: 81-11-706-7876, E-mail: sshimizu-rad@umin.ac.jp

Acknowledgements:

Supported by a grant from the Ministry of Education, Science, Sports, and Culture, Japan (No. 21249065 and No. 0158194) and the Japan Society for the Promotion of Science (JSPS) through the "Funding Program for World-Leading Innovative R\&D on Science and Technology" (FIRST Program).

Conflict of interest: none 


\begin{abstract}
Purpose: Interportal adjustment was applied to patients with prostate cancer using three fiducial markers and two sets of fluoroscopy in a real-time tumor-tracking radiotherapy (RTRT) system. The incidence of table position adjustment required to keep intrafractional uncertainty within $2.0 \mathrm{~mm}$ was investigated in this study.
\end{abstract}

Methods and Materials: The coordinates of the center of gravity of the three fiducial markers were measured at the start of every portal irradiation in intensity-modulated radiotherapy (IMRT) with seven ports. The table position was adjusted to the planned position if the discrepancy was larger than $2.0 \mathrm{~mm}$ in the anterior-posterior (AP), cranio-caudal (CC), or left-right (LR) directions. In total, we analyzed 4541 observations in 20 patients who received $70 \mathrm{~Gy}$ in 30 fractions (7.6 times a day in average).

Results: The incidence of table position adjustment at 10 minutes from the initial set-up of each treatment was $14.2 \%, 12.3 \%$, and $5.0 \%$ of the observations in the AP, CC, and LR directions, respectively. The accumulated incidence of the table position adjustment was significantly higher at 10 minutes compared to that at 2 minutes for $\mathrm{AP}(\mathrm{p}=0.0033)$ and $\mathrm{CC}(\mathrm{p}=0.0110)$ but not LR $(\mathrm{p}=0.4296)$. An adjustment greater than $5 \mathrm{~mm}$ was required at least once in the treatment period in 11 $(55 \%)$ patients.

Conclusions: Interportal adjustment of table position was required in more than $10 \%$ of portal irradiations during the 10-minute period after initial set-up to maintain treatment accuracy within 2.0 $\mathrm{mm}$.

Key words: Radiotherapy, prostate, intra-fraction organ motion, image-guided radiotherapy 


\section{Introduction}

Personalized radiotherapy is required in the era of personalized medicine. Organ motion can be an important patient-specific prognostic factor to improve the therapeutic ratio of radiotherapy. Image-guided radiotherapy (IGRT) is expected to reduce the uncertainty of the localization $(1,2)$.

Frequent displacement of the prostate to the pelvic bony structure has been reported as a problem in the set-up of external radiotherapy of prostate cancer for more than 15 years $(3,4)$. The usefulness of fiducial markers such as radio-opaque materials or electromagnetic devices for the assessment of inter-fraction prostate displacement has also been well established (5-8). Precise re-positioning using an IGRT technique with prostate markers has shown to be useful for reducing the margin of the planning target volume (5-8). The intra-fraction error due to prostate motion was reported to be negligible compared to the inter-fraction set-up error (9).

Recently, however, the intra-fraction motion of the prostate gland has emerged as an important limiting factor when considering margins for intensity-modulated radiotherapy (IMRT), which often requires a treatment time longer than that of conventional treatment. Langen et al. found that for individual patients, the maximal value of displacements $>3 \mathrm{~mm}$ at 5 and $10 \mathrm{~min}$ after initial positioning were $43 \%$ and $75 \%$, respectively (10). Litzenberg et al. reported that for skin-based setup with inclusion of an intra-fraction motion, prostate treatments required average margins of 10.2, 12.5 and $8.2 \mathrm{~mm}$ in the anterior-posterior, cranial-caudal and left-right directions, respectively (11). They suggested that positioning by prostate electromagnetic markers at the start of the treatment fraction reduced these values to $1.8,5.8$, and $7.1 \mathrm{~mm}$, respectively. Most strikingly, they suggested that "interport adjustment" would further reduce margins to an average of 1.4, 2.3, and $1.8 \mathrm{~mm}$. (11). This large difference in the required margin is due to the capability of detection time interval to adjust for intra-fraction prostate motion. Kron et al. evaluated 184 patients who had two orthogonal $\mathrm{x}$-rays with 3 to 30 min between pre- and post-imaging using an on-board $\mathrm{kV}$ imaging system for intra-fraction prostate displacement (12). They found that the mean three-dimensional vector shift between images was $1.7 \mathrm{~mm}$, ranging from 0 to $25 \mathrm{~mm}$. There was a large variation in typical shifts between patients (range $1 \pm 1$ to $6 \pm 2 \mathrm{~mm}$ ) with no apparent trends throughout the treatment course. They concluded that given the variation between patients, a uniform set of margins for all patients might not be satisfactory when high target doses are to be delivered.

To reduce the intra-fraction displacement of the prostate gland during delivery of radiotherapy, we 
have been using implanted fiducial markers and a real-time tumor-tracking radiotherapy (RTRT) system in IMRT for prostate cancer for 10 years. We have adopted "interportal adjustment" of the patient table position during IMRT (13). The preliminary clinical results were encouraging (14). In this study, the incidence of table position adjustment required to keep the intrafractional uncertainty within $2.0 \mathrm{~mm}$ was investigated. The appropriateness of our approach of keeping the target correctly located below the threshold of displacement using interportal adjustment of the table position will be also discussed.

\section{Methods and Materials}

In our treatment protocol for prostate cancer, three 2.0-mm-diameter gold markers were inserted into the prostate gland before computed tomography $(\mathrm{CT})$ for treatment planning. The gold markers were inserted into the clinical target volume (CTV) of the prostate gland; one at the apex of the prostate and two others at the left and right of the base of the gland. Computed tomography of the small pelvis was taken with a $1.0-\mathrm{mm}$ slice thickness and $1.0-\mathrm{mm}$ interval with the patient in the supine position on a flat carbon table. Pinnacle ${ }^{3}$ (Hitachi Medical Co., Tokyo) was used as the three-dimensional radiation treatment planning system (3DRTP). The contours of the prostate gland were defined as CTV, and the positions of the three fiducial markers were determined on 3DRTP using CT images. The coordinates of CTV and the three fiducial markers were determined using the 3DRTP. Planning target volume (PTV) was determined by a three-dimensional expansion of CTV adding a 3-mm margin. Seventy Gy at a D95 of PTV was delivered with step-and-shoot IMRT in 30 fractions in 30 sessions. Seven ports were used in IMRT, and all seven ports were used in each daily treatment.

The RTRT system consists of a conventional 6-MV or 10-MV linear accelerator, two diagnostic $\mathrm{x}$-ray fluoroscopic systems in the linear accelerator room, image processing units, and an image display unit (originally Mitsubishi; changed to Varian Medical Japan Co., Tokyo) $(5,13)$.

The actual position of the markers can be visualized during irradiation. The marker position is transferred from 3DRTP and superimposed on the fluoroscopic image on the display unit of the RTRT system. Details of the calculation of the parallel and rotational set-up error have already been reported (15). In short, the position of the patient can be corrected by adjusting the patient table position by a remote-control bar on the treatment console. When the displacement of the center of 
gravity of the three markers (DCG) exceeds the threshold, the operator can correct the patient table position using the remote control unit. The threshold used in this study was $2.0 \mathrm{~mm}$ in each direction, antero-posterior (AP), cranio-caudal (C-C) and left-right (L-R); thus, if the displacement exceeded $2.0 \mathrm{~mm}$ in any direction, the table position was corrected so that the center of gravity of the three markers would be within $0.1 \mathrm{~mm}$ of its planned position. Therefore, the length (in mm) of the patient table adjustment is equal to the DCG in the body. The table position can be changed in the lateral, vertical and longitudinal directions within an accuracy of $+/-0.1 \mathrm{~mm}$ of the specifications. In our previous study on the RTRT data of 123 set-ups of 5 patients, the random rotational error around the $\mathrm{x}, \mathrm{y}$, and $\mathrm{z}$ axes in the manual set-up was 3.0, 5.1, and 5.0 degrees, respectively. The systematic rotational error around the $\mathrm{x}, \mathrm{y}$, and $\mathrm{z}$ axes in the manual set-up calculated from the 5 patients' data was 3.0, 2.4, and 4.9, respectively (16). Based on these data, we calculated the rotational set-up error around each axis but intentionally did not correct them in this study.

The RTRT system has several options for the frequency of observation. Our system has the option to gate/stop the treatment if the discrepancy from the previous image is over $2 \mathrm{~mm}$, but we would need to expose a diagnostic $x$-ray every $0.033-0.1$ second for this purpose. We decided that it would not be proper to continuously generate diagnostic X-rays during treatment for slow prostate motion. Therefore, we used another option, of consulting a single exposure at the start of every treatment beam portal and intermittently during the beam delivery. Three-dimensional coordinates of the three gold markers were measured with the RTRT system, and the table position was corrected if the DCG was greater than $2.0 \mathrm{~mm}$. For patients in whom displacement was frequently observed, the coordinates of the three markers were measured two times or more during the delivery of one portal irradiation. The position of the patient table was continuously corrected so as not to diverge from the planned position. The time required from the detection to the adjustment of the displacement was usually less than one minute. Thus, the interval between exposures ranged from about 1 to 3 minutes.

The length of the table position adjustment after the initial set-up during the treatment was stored in the data server of the RTRT system. Using the data sets in the server, we could analyze the incidence and magnitude of the interportal requirement of patient table position adjustment after the initial set-up during daily treatment. The incidence should be consistent with the incidence of DCG exceeding the threshold of $2.0 \mathrm{~mm}$ during the irradiation for each port.

In this study, data sets of 20 patients consecutively treated between 2004 and 2008 were used to 
reveal the requirement of interportal patient table position adjustment after the initial set-up during daily treatment to keep the accuracy within $2.0 \mathrm{~mm}$. Patient ages ranged from 55 to 76 years with a median age of 70 . There were 12,4 , and 4 patients with T1N0M0, T2N0M0, and T3N0M0 diseases, respectively. There was no specific regimen for bladder and rectal filling but patients were instructed to void about 1 hour before the time of daily treatment. The study was approved by the institutional ethical committee, and written informed consent was obtained from all patients before the insertion of the markers.

JMP 8.0.1(SAS Institute, Cary, NC, USA) was used for statistical analysis. Statistical significance was tested by the chi-square test. Analysis was performed after treatment for all patients.

\section{Results}

Each patient was treated with 30 sessions, so that data sets of 600 sessions were obtained in total (30 sessions times 20 patients). Data sets of 45 sessions were excluded because of insufficient records or prolonged treatment time due to the general condition of the patients. Consequently, data sets of 555 sessions were used for the analysis. As a result, 4541 observation points were obtained from the 20 patients. The average number of observations per patient was 227.1, and that per session was 7.6.

Figure 1 shows the displacement of the center of gravity of the three gold markers according to the time after the initial set-up at the start of each treatment day. The x-axis shows the time in minutes from the initial set-up, and the y-axis represents the displacement in millimeters for each treatment day. Displacements in the AP, CC and LR directions are plotted on the same graph at the same scale. The displacement was sporadically but definitely larger than $2.0 \mathrm{~mm}$ during the course of IMRT.

The total incidences of patient table position adjustment after the initial setup during treatment was 465 times in total for 30 sessions in the 20 patients. For one patient, the median incidence was 19 times, ranging from 6 times to 68 . The incidences of patient table position adjustment after the initial set-up during treatment are shown in Figure 2. The results show that the incidence of required interportal table position adjustment was as low as $0.5 \%$ within the initial 2 minutes, but its accumulated incidence during daily irradiation was not negligible. Details of the incidence of 
required interportal table position adjustment with 95\% confidence intervals are shown in Table 1. The incidence of table position adjustment was $14.2 \%$ in AP, $12.3 \%$ in CC and 5.0\% in the LR direction, respectively at 10 minutes from the initial set-up of each treatment. The accumulated incidence of table position adjustment was significantly higher at 10 minutes compared to the incidence at 2 minutes in the AP direction $(\mathrm{p}=0.0033)$ and $\mathrm{CC}$ direction $(\mathrm{p}=0.0110)$ but not in the LR direction $(\mathrm{p}=0.4296)$.

Adjustment more than $5 \mathrm{~mm}$ was required at least once in 10 minutes in $7(35 \%)$ patients and at some point in the treatment period in $11(55 \%)$ patients out of the 20 patients entered in this study. If each patient has some characteristics of prostate motion, we may be able to predict the need for interportal adjustment of the table position. We applied the following criteria arbitrarily to stratify the patients into three categories in this study. If displacement exceeded $5 \mathrm{~mm}$ within 10 minutes at least once, the patient was classified into the "large motion" type. Patients who experienced displacement over $5 \mathrm{~mm}$ after 10 minutes but not in the initial 10 minutes were classified as the "increasing" type. If displacement over $5 \mathrm{~mm}$ did not occur even after 10 minutes, the patient was classified as the "steady" type (Figure 3). Applying these criteria, there were 7, 4, and 9 patients in the group of "large motion", "increasing" and "steady" type in our series. For each patient, we investigated whether the grouping from the first 5 fractions placed that patient into the same group as the total 30 fractions. Five of 7 patients with "large motion", 2 of 4 patients with "increasing", and 20 of 9 patients with "steady" type were classified in the same category using the initial 5 fractions.

\section{Discussion}

Recent studies have shown that ultrasound-based systems, in-room CT, in-room kV fluoroscopy, and $\mathrm{CBCT}$ (cone beam computed tomography) are useful in reducing the set-up error for a majority of radiation patients $(17,18,19,20)$. Our method using fiducial markers and two sets of fluoroscopy was also shown to be useful to reduce set-up error compared to the skin-based setting $(5,13,15)$. In this study, we focused on intra-fraction displacement according to treatment time, from the beginning of each treatment session after the daily set-up procedure was finished.

The importance of sporadic intra-fraction prostate motion has been reported in recent years. Kotte et al. analyzed the portal images of 427 patients with stage $\mathrm{T} 3 \mathrm{NxMx}$ prostate carcinoma who received IMRT combined with position verification with fiducial markers with the irradiation time of 
5-7 $\mathrm{min}(20)$. In $66 \%$ of the treatment fractions, a motion outside the range of $2 \mathrm{~mm}$ was observed, with $28 \%$ outside the range of $3 \mathrm{~mm}$. They found that intra-fraction motion caused position uncertainty with systematic errors $(\Sigma)$ to $<0.6 \mathrm{~mm}$ and random errors $(\sigma)$ to $<0.9 \mathrm{~mm}$, and suggested a lower limit of $2 \mathrm{~mm}$ for margins with online position correction at the start of irradiation. We also found that the intra-fraction displacement was usually as small as $2 \mathrm{~mm}$ on average during the initial 2 minutes. However, the displacement became larger according to the elapsed treatment time after the start of radiotherapy in our series. A similar trend was observed in recent studies in which patients had treatment times longer than 5 minutes $(10,22)$. Thus, among patients expected to have radiotherapy lasting longer than 2 minutes, careful observation during the delivery of radiotherapy with interportal adjustment may be useful for a small but definite number of patients.

In this study, interportal adjustment of the patient table combined with usage of three implanted markers and two sets of fluoroscopy was shown to be effective in maintaining the accuracy of the prostate position. The benefit of quick estimation of prostate displacement using the RTRT system was apparent considering the minimal elongation of the treatment time. Similar mid-session adjustment of table position has been reported using a robotic linear accelerator for hypofractionated radiotherapy of the prostate, which often requires 50 to $70 \mathrm{~min}$ for one treatment session (23). Those authors found that when sporadic prostate movements greater than $5 \mathrm{~mm}$ were present in any one direction, significant changes in the dose volume histogram could be detected. Compared to their stereotactic hypofractionated radiotherapy, our protocol has a shorter daily treatment time. However, step-and-shoot IMRT often requires $10 \mathrm{~min}$, which is still long enough for an intra-fraction prostate motion larger than $5 \mathrm{~mm}$ to occur.

Litzenberg et al. have estimated that mid-session adjustment would reduce margins to an average of 1.4, 2.3, and $1.8 \mathrm{~mm}(11)$. Their results are consistent with Nederveen et al.'s study suggesting that a 1- to 2-mm margin is sufficient for intra-fraction displacement providing that position verification is performed at time intervals of 2 to $3 \mathrm{~min}$ (7). We confirmed that the margin for prostate motion can be significantly reduced using our interportal adjustment technique. The margin for internal organ motion was kept at $2 \mathrm{~mm}$ for each direction in our protocol.

It is still not certain whether we should use real-time tracking of the prostate markers during the delivery of radiotherapy as RTRT for lung cancers (24), and permit irradiation only when the fiducial markers are within the gating window. Litzenberg et al. suggested that 2 of their 11 patients would have benefited from continuous target tracking and threshold-based intervention from their analysis 
of intra-fraction organ motion (11). Nederveen et al. found marker displacements as large as $9.5 \mathrm{~mm}$ in one fraction and suggested the need for frequent verification in some patients (7). We also experienced several patients other than the 20 patients in this study for whom the prostate position was so unstable that real-time tracking of the marker and gated irradiation was used. The amount of motion of the prostate is far different than that from respiratory motion, probably due to the motion of the gas in the rectum. On the other hand, a large proportion of patients experienced not so large displacement during their irradiation. Appropriate criteria are required to use real-time tracking of the marker and threshold-based intervention.

We found at least three types of patients in terms of internal prostate motion. If we could predict which patients are steady types, we would not require any online monitoring of the prostate motion during delivery of their radiotherapy. Likewise, if a patient could be pre-identified as a large-motion type, frequent monitoring or even real-time tracking of the marker position could be used to reduce the risk of adverse effects and local relapse. For patients in the increasing-motion type, modest monitoring of the marker would be appropriate. In our preliminary analysis in this study, we found that we can detect patients with large motion with considerable probability from the observation of the first 5 fractions. However, the distinction between the increasing and steady types seemed to be difficult. These types were arbitrarily determined in this study, and the distinction requires further analysis.

The shortcomings of this study are as follows. First, frequent observation of the markers using diagnostic fluoroscopy increases the patients' radiation exposure. However, since a couple of orthogonal x-ray static images are sufficient to measure the displacement of the prostate gland in the interportal adjustment, the total amount of exposure is estimated to be negligible with seven-portal IMRT. Making position corrections using RTRT system, as other IGRT devices, can reduce CTV-PTV margin, which might otherwise exceed that actually required, and therefore reduce the dose around the CTV (25).

Secondly, a treatment time of more than $10 \mathrm{~min}$ for IMRT may be too long in the era of high-dose-rate external radiotherapy. Our results may be regarded as data to support the appropriateness of developing a high-dose-rate external radiotherapy system with a short treatment time. Aznar et al. reported when the volumetric modulated arc therapy was used to treat prostate cancer patients, it required less than 2 minutes of beam-on time per treatment (26). In our study, within 2 minutes after initial patient setup for daily treatment, the movement of the prostate was 
limited. Thus, a faster treatment is suitable for avoiding excursion or drifts of the target when an intra-fraction adjustment is not used. On the other hand, spot scanning particle beam therapy and intensity-modulated proton beam therapy (IMPT) is now becoming available to reduce the low-dose large-area irradiation in IMRT and neutron contamination in conventional proton therapy. These new techniques would require more than several minutes with the expectation for higher accuracy in patient positioning. Our results suggest that these "high-tech" methods should match the requirement for "interportal" adjustment of the treatment position to accomplish their goal.

In conclusion, the displacement during 10 minutes was significantly larger than the displacement during the initial 2 minutes. The probability of displacement of more than $2.0 \mathrm{~mm}$ is under $0.5 \%$ in the initial 2 minutes in the AP, CC, and LR directions, respectively. However, without interportal adjustment of the patient table, intra-fraction displacement may not be negligible in treatments longer than 2 minutes. Interportal adjustment of table position during the 10 minutes after initial set-up was required in more than $10 \%$ of portal irradiations to maintain treatment accuracy within $2.0 \mathrm{~mm}$. Implantation of three fiducial markers and interportal adjustment of the patient table with the RTRT system was shown to be useful in maintaining the intra-fraction displacement within the predetermined range of $2.0 \mathrm{~mm}$ for localized prostate cancer. 


\section{References}

1. Verellen D, De Ridder M, Linthout N, et al. Innovations in image-guided radiotherapy. Nat Rev Cancer 2007; 7: 949-960.

2. Shirato $\mathrm{H}$, Organ motion in image-guided radiotherapy: lessons from real-time tumor-tracking radiotherapy. Int J Clin Oncol 2007; 12: 8-16.

3. van Herk M, Bruce A, Guus Kroes AP, et al. Quantification of organ motion during conformal radiotherapy of the prostate by three dimensional image registration. Int J Radiol Oncol Biol Phys 1995; 33: 1311-1320.

4. Crook JM, Raymond Y, Salhani D, et al. Prostate motion during standard radiotherapy as assessed by fiducial markers. Radiother Oncol 1995; 37(1): 35-42.

5. Shimizu S, Shirato H, Kitamura K, et al. Use of an implanted marker and real-time tracking of the marker for the positioning of prostate and bladder cancers. Int J Radiat Oncol Biol Phys 2000; 48(5): 1591-7.

6. Wu J, Haycocks T, Alasti H, et al. Positioning errors and prostate motion during conformal prostate radiotherapy using on-line isocentre set-up verification and implanted prostate markers. Radiother Oncol 2001; 61(2): 127-133.

7. Nederveen AJ, van der heide UA, van Moorselaar RJ, et al. Measurements and clinical consequences of prostate motion during a radiotherapy fraction. Int J Radiat Oncol Biol Phys 2002; 53(1): 206-14.

8. Schallenkamp JM, Herman MG, Kruse JJ, et al. Prostate position relative to pelvic body anatomy based on intraprostatic gold markers and electronic portal imaging. Int J Radiat Oncol Biol Phys 2005; 63(3): 800-811.

9. Huang E, Dong L, Chandra A, et al. Intrafraction prostate motion during IMRT for prostate cancer. Int J Radiat Oncol Biolo Phys 2002; 53(2): 261-268.

10. Langen KM, Willoughby TR, Meeks SL, et al. Observations on real-time prostate gland motion using electromagnetic tracking. Int J Radiat Oncol Biol Phys 2008; 71(4): 1084-90.

11. Litzenberg DW, Balter JM, Hadley SW, et al. Influence of intrafraction motion on margins for prostate radiotherapy. Int J Radiat Oncol Biol Phys 2006; 65(2): 548-53.

12. Kron $\mathrm{T}$, Thomas J, Fox $\mathrm{C}$, et al. Intra-fraction prostate displacement in radiotherapy estimated from pre- and post-treatment imaging of patients with implanted fiducial markers. Radiother Oncol 2010; Feb 26, Epub ahead of print. 
13. Shirato H, Oita M, Fujita K, et al. Three-dimensional conformal setup (3D-CSU) of patients using the coordinate system provided by three internal fiducial markers and two orthogonal diagnostic X-ray systems in the treatment room. Int J Radiat Oncol Biol Phys 2004; 60(2): 607-12.

14. Kitamura K, Shirato H, Shinohara N, Reduction in acute morbidity using hypofractionated intensity-modulated radiation therapy assisted with a fluoroscopic real-time tumor-tracking system for prostate cancer: preliminary results of a phase I/II study. Cancer J 2003; 9(4): 268-276.

15. Shirato H, Shimizu S, Kunieda T, et al. Physical aspects of a real-time tumor-tracking system for gated radiotherapy. Int J Radiat Oncol Biol Phys 2000; 48(4): 1187-95.

16. Fujita K, Shirato H, Kitamura K, et al. Three-dimensional conformal set-up of prostate cancer by adjustment of actual clinical target volume (CTV) to virtual CTV using three fiducial markers and fluoroscopic real-time tracking system. Int J Radiat Oncol Biol Phys 2001; 51(3): 384.

17. Wong J, Grimm L, Uematsu M, et al. Image-guided radiotherapy for prostate cancer by CT-linear accelerator combination: prostate movements and dosimetric considerations. Int $\mathrm{J}$ Radiat Oncol Biol Phys 2005; 61(2): 561-569.

18. Adamson J, Wu Q. Prostate intrafraction motion evaluation using $\mathrm{kV}$ fluoroscopy during treatment delivery: a feasibility and accuracy study. Med Phys 2008; 35(5): 1793-806.

19. Adamson J, Wu Q. Prostate intrafraction motion assessed by simultaneous kilovoltage fluoroscopy at megavoltage delivery I: clinical observations and pattern analysis Int J Radiat Oncol Biol Phys 2010; 78(5): 1563-70.

20. Pinkawa M, Pursch-Lee M, Asadpour B, et al. Image-guided radiotherapy for prostate cancer. Implementation of ultrasound-based prostate localization for the analysis of inter- and intrafraction organ motion Strahlenther Onkol 2008; 184(12): 679-85.

21. Kotte A, Hofman P, Lagendijk J, et al. Intrafraction motion of the prostate during external-beam radiation therapy: analysis of 427 patients with implanted fiducial markers. Int J Radiat Oncol Biol Phys 2007; 69(2): 419-425.

22. Xie Y, Djajaputra D, King CR, et al. Intrafractional motion of the prostate during hypofractionated radiotherapy. Int J Radiat Oncol Biol Phys 2008; 72(1): 236-46.

23. Hossain S, Xia P, Chuang C, et al. Simulated real time image guided intrafraction tracking-delivery for hypofractionated prostate IMRT. Med Phys 2008; 35(9): 4041-8. 
24. Onimaru R, Fujino M, Yamazaki K, et al. Steep dose-response relationship for stage I non-small-cell lung cancer using hypofractionated high-dose irradiation by real-time tumor-tracking radiotherapy. Int J Radiat Oncol Biol Phys 2008; 70(2): 374-81.

25. Kron T, Wong J, Rolfo A, et al. Adaptive radiotherapy for bladder cancer reduces integral dose despite daily volumetric imaging. Radiother Oncol 2010; 97(3): 485-7.

26. Aznar MC, Petersen PM, Logadottir A, et al. Rotational radiotherapy for prostate cancer in clinical practice. Radiother Oncol 2010; 97(3): 480-4. 


\section{Figure Legends}

\section{Figure 1.}

The displacement of the center of gravity of the three gold markers (DCG) according to time after the initial set-up at the start of each treatment day in 20 patients. The $\mathrm{x}$-axis shows the time in minutes from the initial set-up and the $y$-axis represents the amount of the displacement in $\mathrm{mm}$ for each treatment day. Displacements in the antero-posterior (AP), cranio-caudal (CC) and left-right (LR) directions are plotted on the same scale.

\section{Figure 2.}

Incidences of patient table position adjustment after the initial set-up during the treatment. The length of the table position adjustment was stratified in 2-mm intervals. The incidence of adjustment was stratified in 2-minute intervals after the initial set-up. The figure shows the cumulative incidence of displacement at 2, 4, 6, 8, 10 minutes, and thereafter.

\section{Figure 3.}

The displacement of the center of gravity of the three markers (DCG) in three patients who were stratified into "large motion", "increasing", and "steady" types, respectively.

\section{Table 1.}

Means and 95\% confidence intervals of the distribution of incidence of prostate displacement. Each cell is gray-coded according to probability: $<0.5 \%$, white; $0.5 \%-5 \%$, light gray; $\geq 5 \%$, gray. 


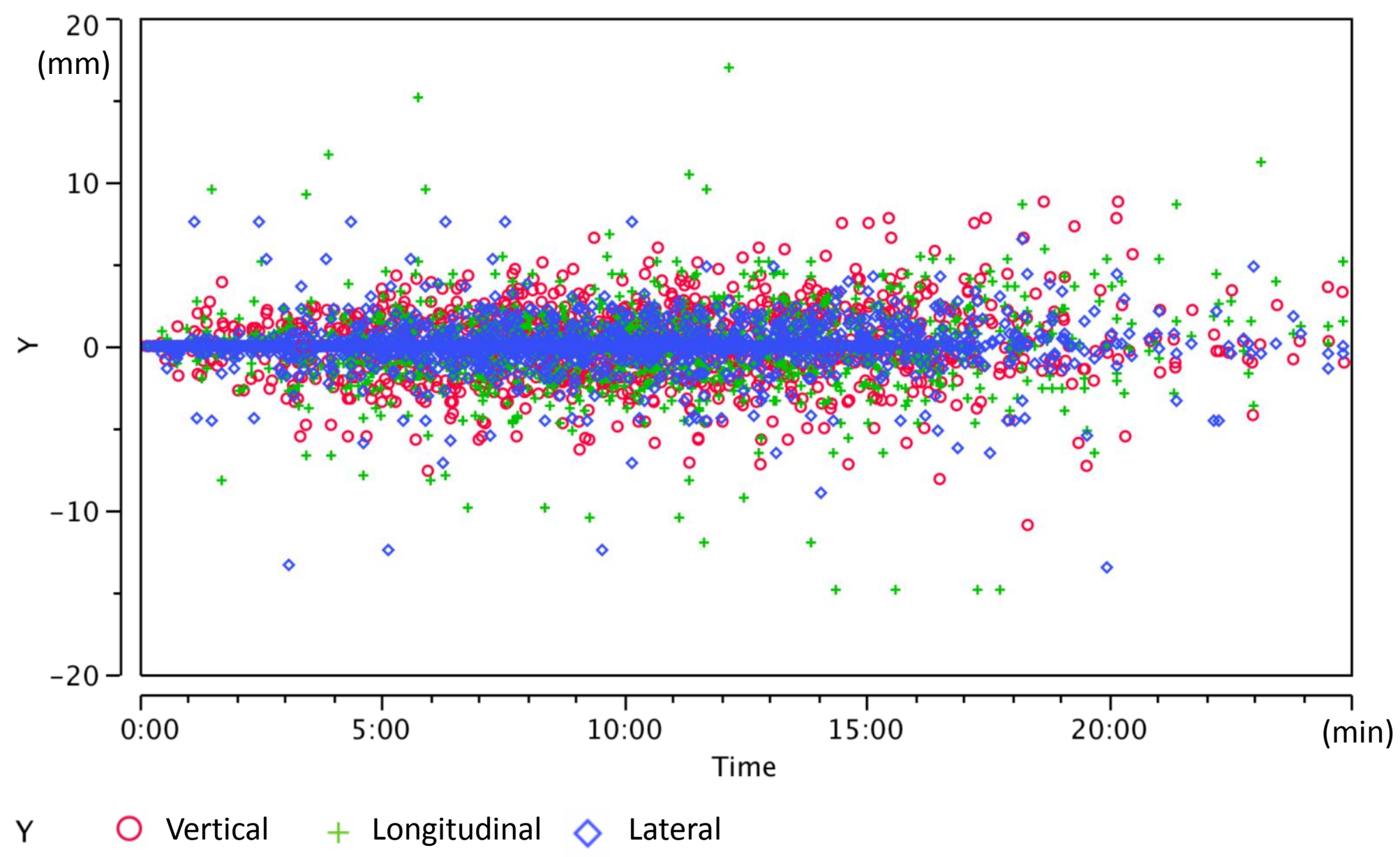

Figure 1 


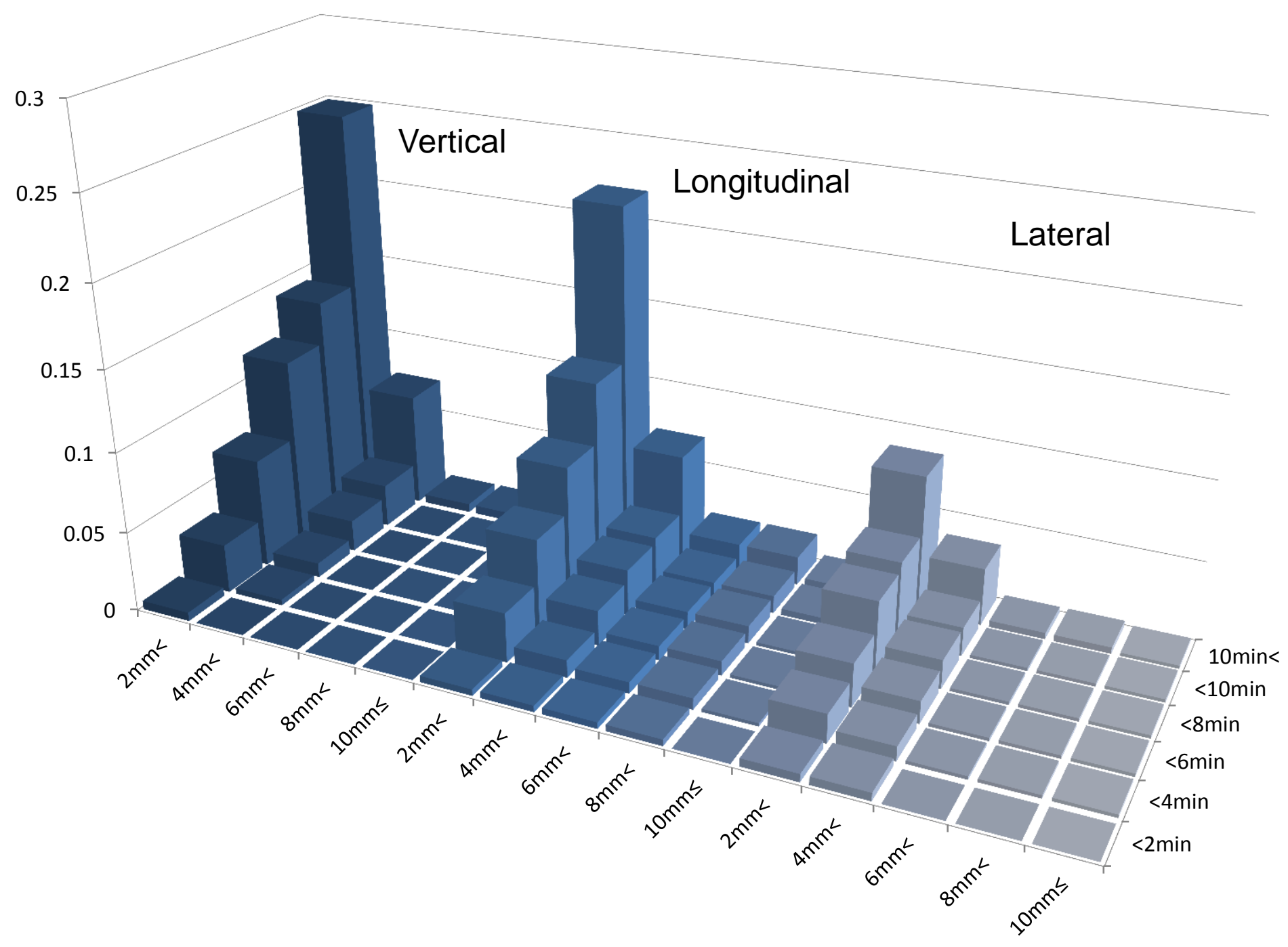

Figure 2 

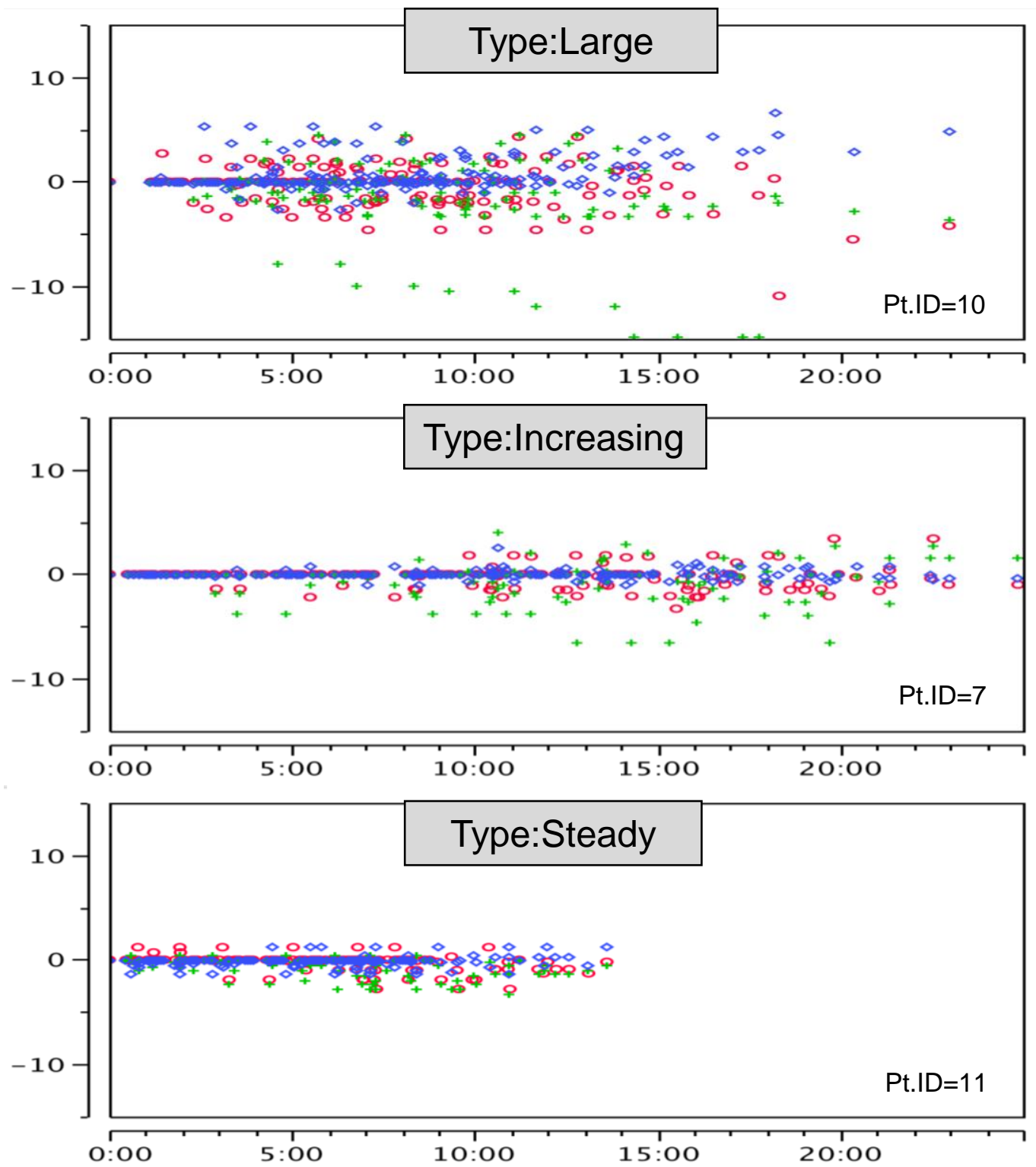

$\bigcirc$ Vertical + Longitudinal $\diamond$ Lateral

\section{Figure 3}




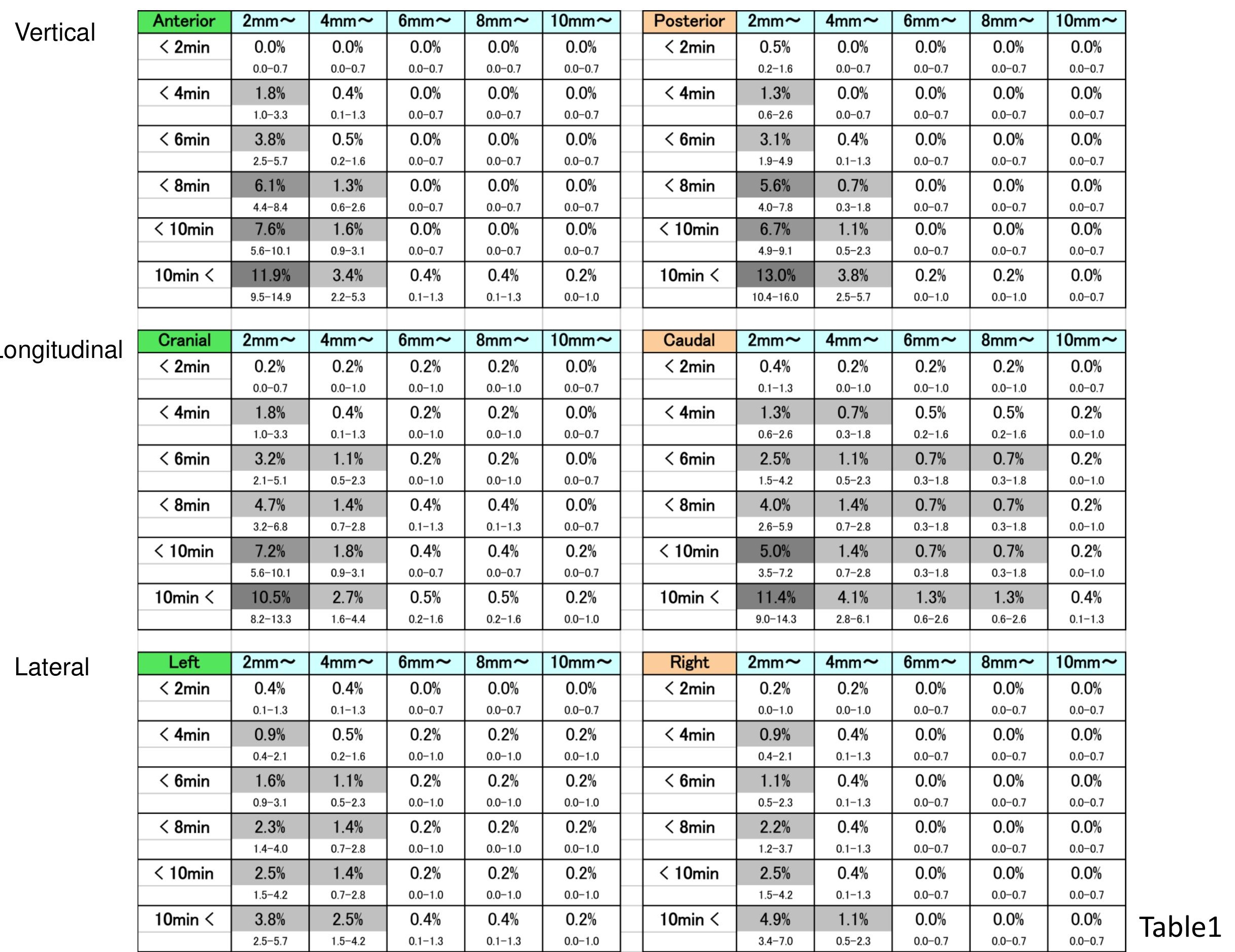

